\title{
Polarization and spectral filter arrays based on sub-wavelength structures in CMOS
}

Junger, Stephan; Tschekalinskij, Wladimir; Verwaal, Nanko; Weber, Norbert

Fraunhofer Institute for Integrated Circuits IIS

Am Wolfsmantel 33, 91058 Erlangen, Germany

\begin{abstract}
Polarization and spectral selective sensors can be implemented by using sub-wavelength gratings and hole arrays in metal films, respectively. In this paper we demonstrate the fabrication of so-called wire grid polarizers and hole arrays for color sensing using complementary metal-oxide semiconductor (CMOS) processes. The simulation of the structures was based on the finite-difference time-domain (FDTD) method, and the layout of the nanostructures was generated using the work flow of integrated circuits. A polarization image sensor was designed and demonstrated, and multispectral sensors were implemented using nanostructures with different spectral filter performances on a single chip.
\end{abstract}

\section{Introduction}

In order to achieve polarization or spectral selectivity, conventional detectors and detector arrays require additional optical elements. Polarization filters, prisms, or beam splitters are used for polarization sensing, color filters or dispersive elements like diffraction gratings for color and multispectral sensors. Especially a pixel-wise alternating filter array for image sensors is difficult to fabricate and align and only standard filter arrangements like the red/green/blue filter mosaic of the well known Bayer pattern for color image sensors are established.

\section{Methods}

We demonstrate a different approach, where optical nanostructures - combined with underlying photodiodes - are used for filtering the light (fig.1 ). Sub-wavelength gratings act as wire grid polarizers and sub-wavelength hole arrays are used as spectral filters. The optical properties of these nanostructures are well understood, but fabrication is usually done using technologies like electron beam lithography or focused ion beam milling. As opposed to that, we used deep submicron CMOS (complementary metal-oxide-semiconductor) manufacturing processes, allowing for high-volume fabrication of photodiodes including optical filters by patterning the metal layers ("More than Moore").
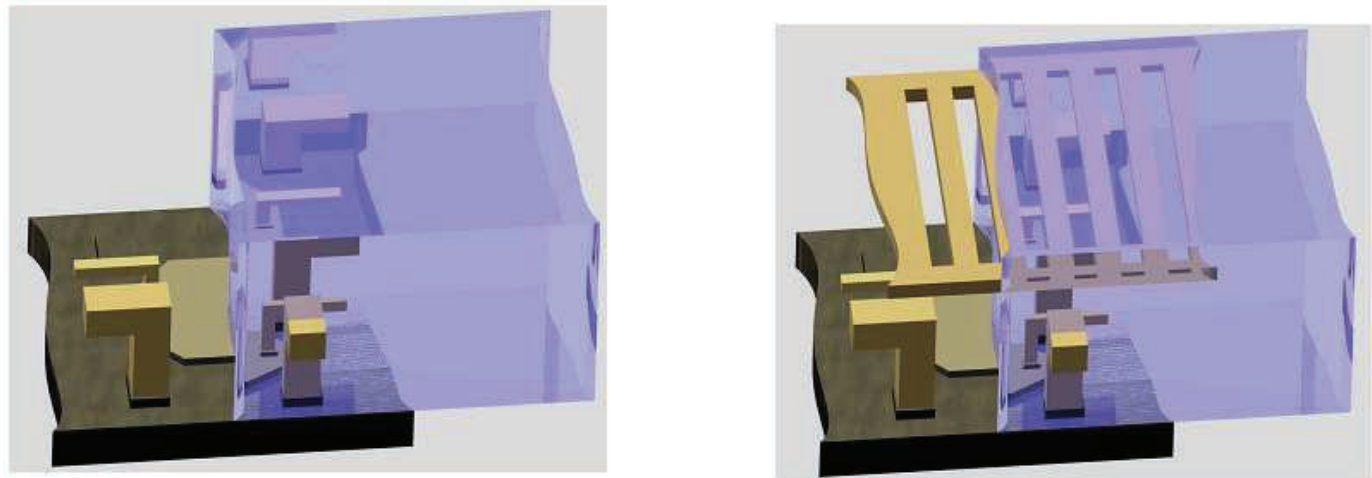

Fig. 1: Cross section of a conventional CMOS photodiode (left) and a photodiode with nanostructures (right)

The simulation of the nanostructures was based on the finite-difference time-domain (FDTD) method, using the software packages MEEP from MIT and the commercial software package OptiFDTD. FDTD is based on a discrete decomposition of the Maxwell equations into spatial and temporal steps, using numerical techniques to calculate the evolution of the electromagnetic fields in time. Provided that precise material models and adequate spatial resolutions are used for simulation, FDTD yields reliable results matching numerous experiments. 
After design and simulation, the layout was generated during the IC design flow. Several test chips were fabricated using the CMOS processes of different semiconductor foundries and the successful patterning of the nanostructures was verified by scanning electron microscopes. Each test chip comprises of photodiodes covered by different nanostructures; photodiodes without nanostructures are used as a reference. The spectral transmission of the nanostructures is obtained by calculating the ratio of the absolute spectral response of test photodiodes and reference photodiodes.

\section{Results}

Fig. 2 shows the measured performance of a grating with a period of $400 \mathrm{~nm}$, and the expected polarizing effect is evident for wavelengths beyond $800 \mathrm{~nm}$, where TE-polarized light is suppressed efficiently and the transmission of TM-polarized light is approximately $30 \%$.

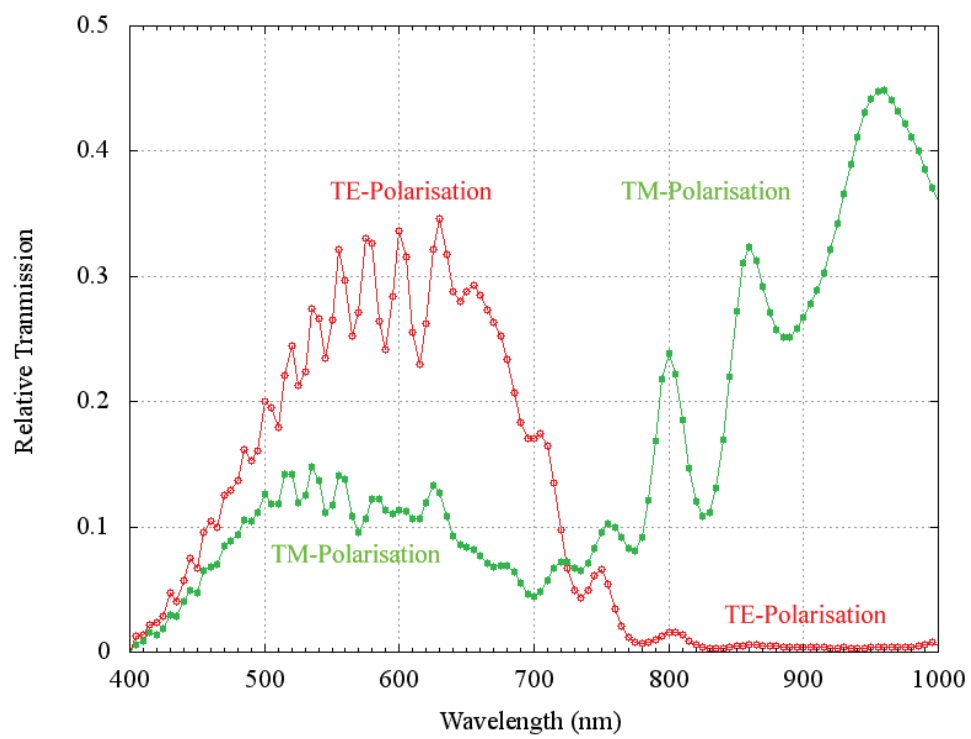

Fig. 2: Measured transmission of a polarization selective grating with a period of $400 \mathrm{~nm}$

This concept can be applied to single polarization sensors, and also to pixel arrays where different orientations of the gratings allow the detection of polarized light for polarization. Figure 3 shows the arrangement of such a pixel and polarizer array.

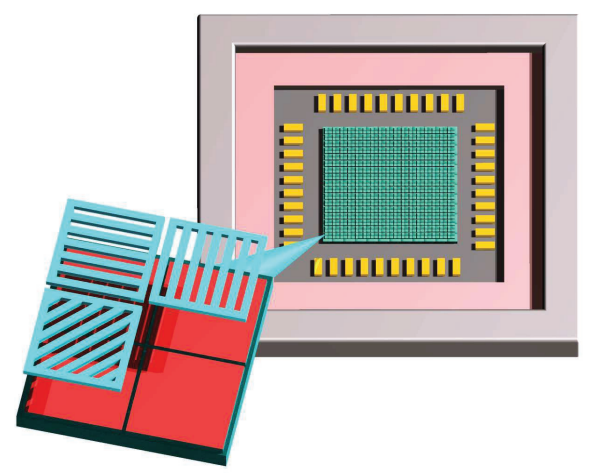

Fig. 3: Concept of the CMOS polarization image sensors

This concept was demonstrated by fabricating a polarization image sensors with an array of $560 \times 254$ pixels with a size of $6 \mu \mathrm{m}$, covered by an array of polarization filters. The smallest period of the grating allowed by the design rules of the process was $400 \mathrm{~nm}$, yielding a good polarization selectivity in the near infrared above $800 \mathrm{~nm}$. 
In order to achieve image acquisition, the polarization image sensor was evaluated in a prototype camera setup showing stress birefringence of a polymer slab (fig. 4). The Stokes parameters - describing the polarization state of the light - are calculated from the different pixel data and are shown as false colors.

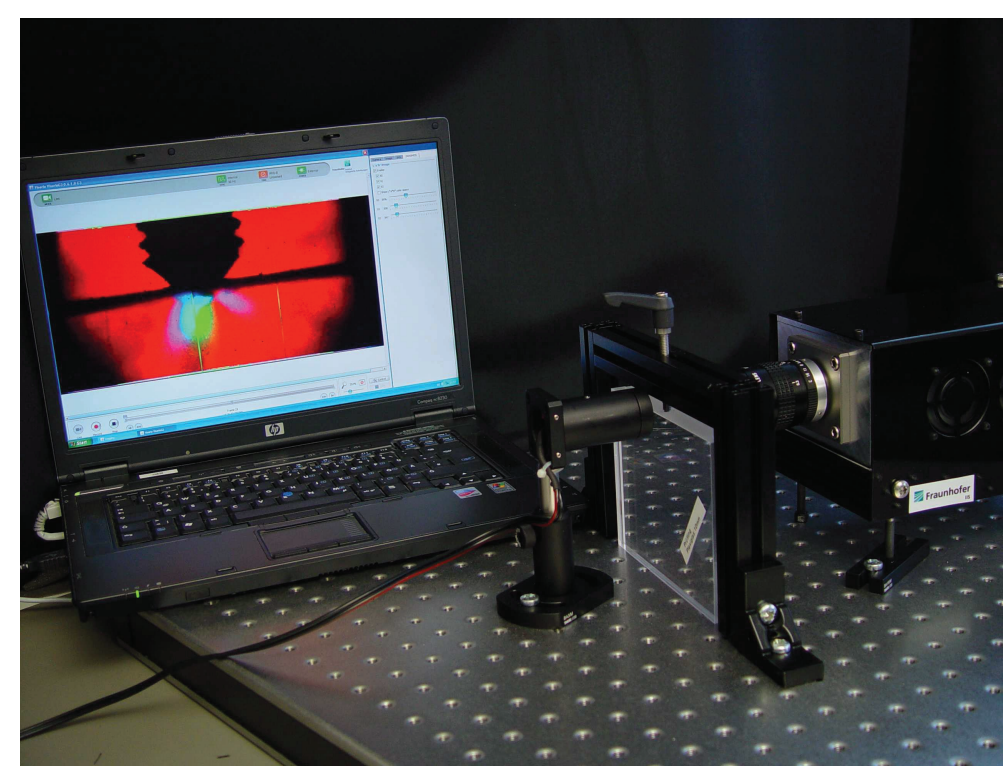

Fig. 4: Demonstration setup for polarization imaging showing stress birefringence of a polymer slab

As opposed to polarization filters, where grating structures are used, color filters were realized based on sub-wavelength hole arrays. Several test chips were processed with varying nanostructures for color and multispectral sensing. Fig. 5. shows one of these chips, the size of each photo diode is $300 \times 300 \mu \mathrm{m}^{2}$, the total chip size is approximately $8 \times 7 \mathrm{~mm}^{2}$.

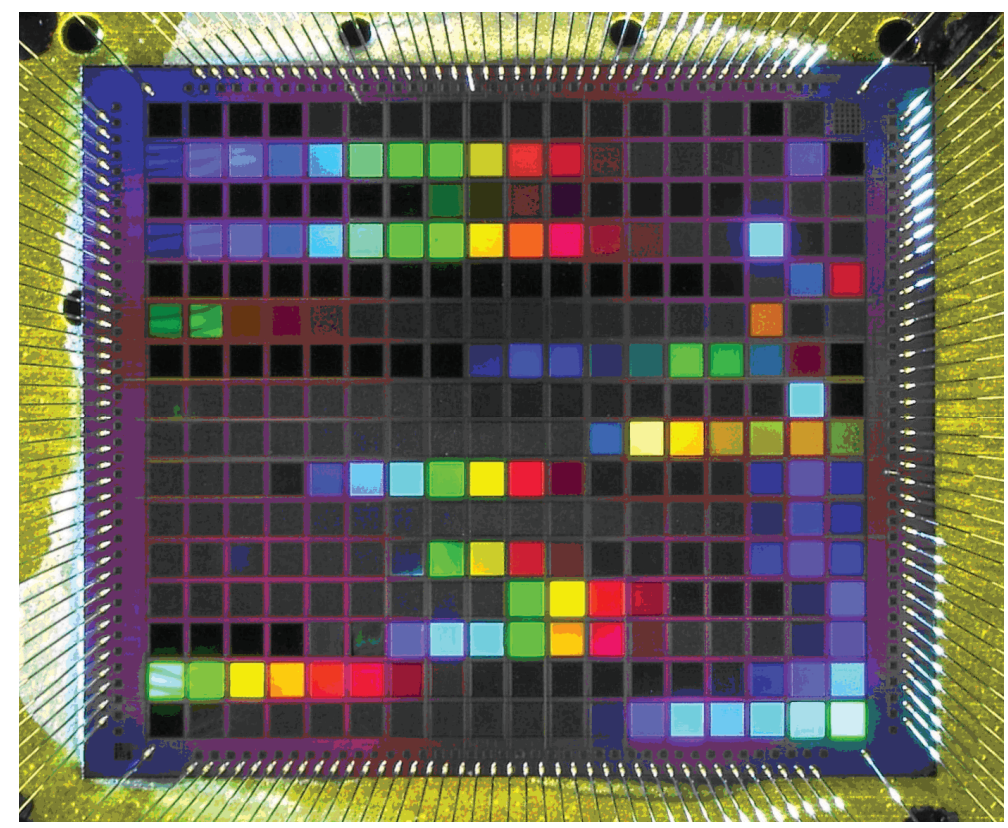

Fig. 5: Test chip with different nanostructures and white light illumination 
Spectral filtering is achieved by nanohole arrays with different geometry, an example for a band-pass filter with a peak wavelength of $520 \mathrm{~nm}$ and a peak transmission of $50 \%$ is given in fig. 6 .

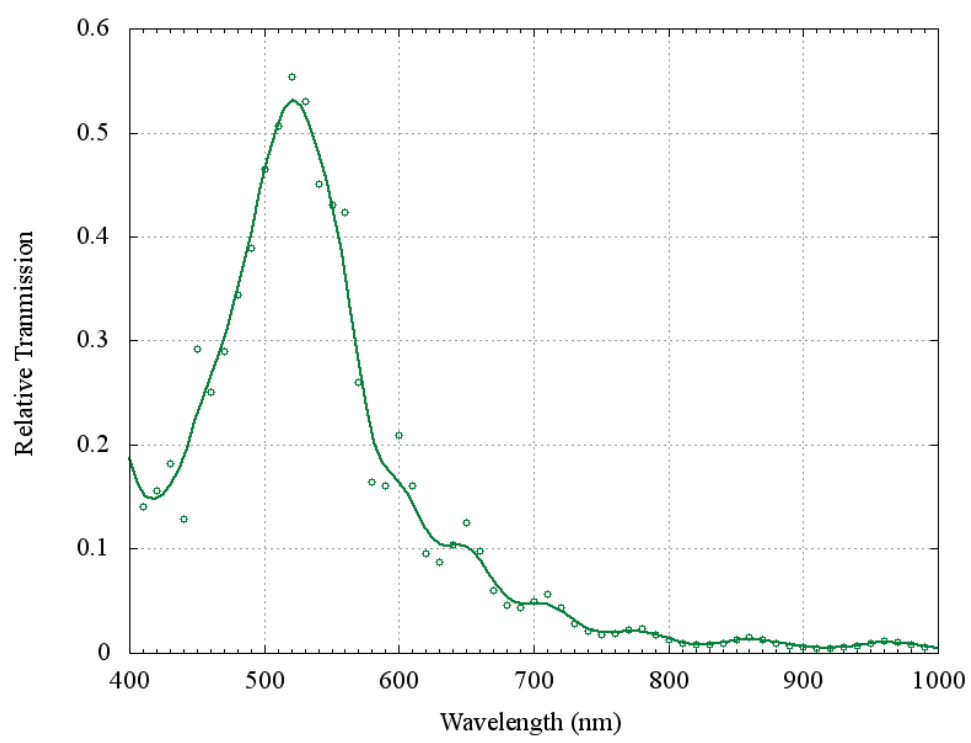

Fig. 6: Measured spectral transmission of a band pass filter for green light $(520 \mathrm{~nm})$

The peak wavelength can be tuned by modifying the lateral geometry of the nanostructures at constant layer thickness, and this possibility of tailoring the band pass filter is an important feature for implementing many photo diodes with different spectral sensitivities on a single chip for on-chip color and multispectral sensors. Fig. 7 shows a band pass filter for yellow light $(590 \mathrm{~nm})$ and a peak transmission of $35 \%$.

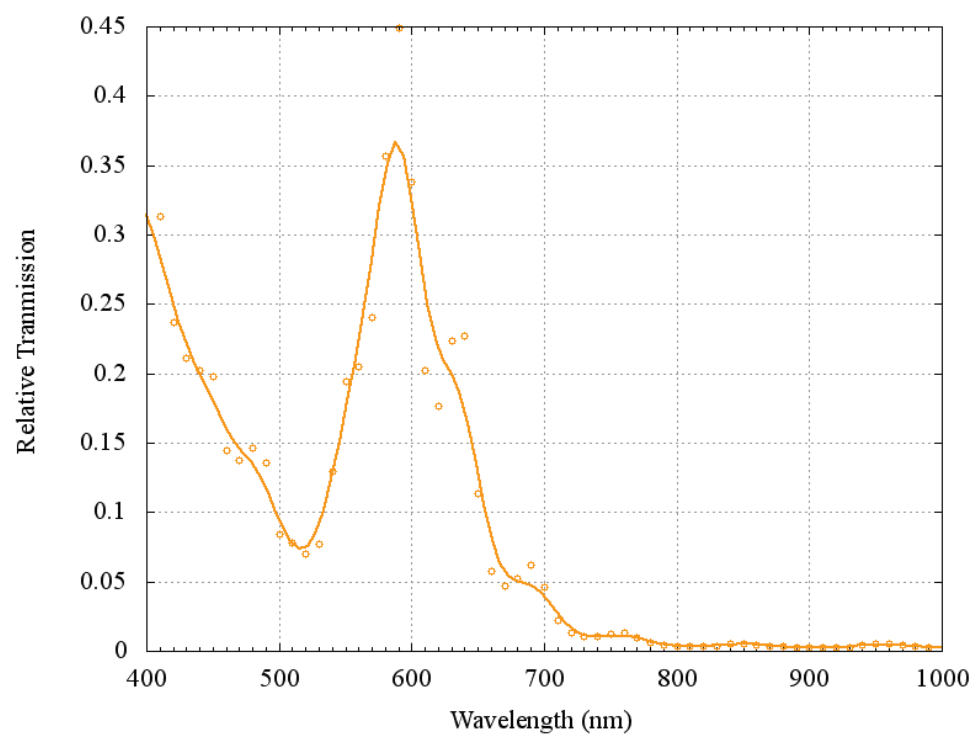

Fig. 7: Measured spectral transmission of a nanostructure tailored for a peak transmission for yellow light 


\section{Conclusions}

In this paper we demonstrated the feasibility to realize wire grid polarizers and sub-wavelength hole arrays for color filtering using the metal layers of a CMOS process. Gratings can be applied to photodiodes, but also to the pixels of an image sensor, and a polarization image sensor was demonstrated. Spectral filtering based on hole arrays can be tailored by varying the geometry of the subwavelength structure, and different band pass filters were fabricated. The metallic nanostructures were simulated by the FDTD method, and the chip layout was generated during the work flow of integrated circuit design. The demonstrated on-chip polarization and spectral filters can be applied to polarimetry, polarization imaging, spectral monitoring of LEDs, color detection, and multispectral sensing.

\section{Literature}

[1] Seh-Won Ahn et al.; Fabrication of a $50 \mathrm{~nm}$ half-pitch wire grid polarizer using nanoimprint lithography; Nanotechnology 16; pp. 1874-1877, 2005

[2] P. B. Catrysse, B. A. Wandell; Integrated color pixels in $0.18 \mu \mathrm{m}$ complementary metal oxide semiconductor technology; J. Opt. Soc. Am., Vol. 20, No. 12 Dezember, pp. 2293-2306, 2003

[3] Ebbesen, T. W., Lezec, H. J., Ghaemi, H. F., Thio, T., and Wolff, P. A.; Extraordinary optical transmission through sub-wavelength hole arrays; Nature (London) 391, 667, 1998

[4] Allen Taflove, Susan C. Hagness; Computational Electrodynamics: The Finite-Difference TimeDomain Method; Artech: Norwood, MA, 2000 